heart failure patients within the specialist palliative care services at the hospice.

The service had six core aims:

1. To establish a heart failure clinic at the hospice;

2. To develop a heart failure monthly meeting focusing on reablement and including planned sessions on fatigue management, breathlessness management etc;

3. To develop a new service planning focus group of heart failure patients and carers co-designing and co-producing future services to meet their identified needs;

4. To support the heart failure team to develop and run a heart failure multi-disciplinary team meeting in partnership with palliative care colleagues;

5. To further develop peer learning between heart failure specialist and specialist palliative care teams;

6. To establish a conversational training programme for primary and secondary health care colleagues focusing on advance care planning and prognostication in heart failure.

The project achieved five of our six objectives during the 18 month period. In addition it produced resources as a legacy to the project including patient designed leaflets for individuals being referred to the hospice, patient designed leaflets for their family and carers about allowing them to try things independently even if it takes longer to complete a task and a DVD on mastering breathlessness.

Both quantitative and qualitative data demonstrated positive outcomes, which have resulted in substantive funding from the hospice to continue the service.

The project team were invited to present the work orally at the 9th Annual Heart Failure Conference in Glasgow in March 2019, generating national interest in this partnership working.

\section{P-189 DOES CHANGE ACTUALLY MEAN CHANGE? HOW DID A NON-PALLIATIVE SERVICE REVIEW INFLUENCE AND BENEFIT THE PALLIATIVE LYMPHOEDEMA SERVICE?}

Karen Tudge. Dorothy House Hospice Care, Winsley, UK

\subsection{6/bmjspcare-2019-HUKNC.211}

In January 2018 Dorothy House Hospice Care commenced a service cost project to establish baseline figures and activity that would influence the future negotiations with the Non-Palliative Lymphoedema service. This exercise highlighted that the non-palliative service was not cost effective and was ineffective in being responsive to new referrals and follow-ups.

Due to ineffective diary management, lack of clinics and lack of time to engage with external referrers both the Non Palliative and Palliative Service were affected and response to referrals were poor.

Benefits of service review

- Opportunity to fully cost out a non-palliative service and ensure the service model is cost effective and ensure transparency with contracting and costings;

- Opportunity to redesign services to enable quality improvement to be part of continuous improvement of services;

- Opportunity to integrate Palliative and Non Palliative Services together to utilise fully the skills of the wider team;
- Provide a more responsive and consultative model of care to palliative patients.

Issues

- Conflict of historic model of care and changing processes within provider and commissioning service;

- Withdrawal of non-palliative contract meant reduction in team numbers and staff consultation proceeded;

- Historic expectations of hospice providing services based on thought and not need;

- Conflict about care provision to palliative vs non-palliative patients;

- Loss of experienced staff;

- Lack of non-palliative contract knowledge by external team members created uncertainty within core palliative services.

The future Through valuation and redesign/cost modelling of the non-palliative contract we have been able to fully cost out and design a service that is responsive, safe and provides quality care in a holistic manner. Through remodelling of the service we have been able to create additional time to support existing staff within the hospice to understand palliative lymphoedema and provide support and guidance to the patients etc. based on the lymphoedema service.

\section{P-190 IMPLEMENTING A BESPOKE AND DEDICATED TRIAGE PROCESS FOR REFERRALS TO A HOSPICE LYMPHOEDEMA CLINIC}

Lynda Parrish, Lisa Cresswell, Elaine Green, Jane Tyler, Rebecca Chambers. Ashgate Hospicecare, Chesterfield, UK

\subsection{6/bmjspcare-2019-HUKNC.212}

Aim To improve the patient experience for anyone diagnosed with cancer or a life limiting illness who has been referred to the hospice for lymphoedema treatment.

To provide a more personalised, responsive service, allowing patients a more appropriate appointment in their preferred clinic location to reassure them and provide education at an earlier time point.

Description The team developed a bespoke triage process and improved the patient experience by mapping the existing lymphoedema service referral process and developing clear aims, objectives and key performance indicators.

A new process and criteria was designed, tested, implemented and evaluated with the aim of improving the response of the first contact. We provide patients with an earlier opportunity to access other hospice services to improve their whole experience.

All referrals are triaged by a lymphoedema specialist by telephone within one week of receiving the referral and a bespoke template is then used to identify the level of need and to determine the priority of care required.

Evaluation The results have shown this change in service delivery has dramatically improved the process of referrals and has had a positive benefit to the patient and carer experience by addressing all forms of physical and emotional distress as demonstrated in our Service Quality Health Survey.

This has reduced the number of inappropriate referrals and the team has been able to signpost patients to other hospice services in a more timely way, thereby reducing waiting time, multiple telephone contacts and unnecessary visits and enabling a more personalised and responsive service. 
It has led to an improvement in patients' knowledge and understanding of their condition, empowering them to commence basic treatment prior to the first appointment.

\section{P-191 LIVING WELL: AN EARLY ACCESS PROGRAMME FOR PEOPLE LIVING WITH MND}

Yvonne Dunn. Marie Curie Hospice Newcastle, Newcastle Upon Tyne, UK

10.1136/bmjspcare-2019-HUKNC.213

Marie Curie Hospice Newcastle, which provides care and support to people who have received a terminal diagnosis and their carers, embarked on a service improvement project with a view to improving the experience of people living with Motor Neurone Disease (MND) at an early stage of their diagnosis. This project was delivered in partnership with the Motor Neurone Disease Association (MND Association), and with their support we have developed the group, Living Well: an early access programme for people living with MND.

Anecdotal evidence suggests patients diagnosed with MND have limited access to support networks at the early stages of their condition. Many patients also report that they prefer not to think too far ahead as a coping strategy to this changing condition, which can lead them to decline engagement with any such services that may be available.

The group began in September 2017 and has offered practical advice on living well with MND including basic moving and handling techniques; education sessions from a range of professionals including dietician, speech and language therapist and benefits advisor; and received complementary therapies such as massage and reiki.

The group has been running for over eighteen months. We are continually reflecting, reviewing and implementing change as appropriate. We have received fantastic user feedback from patients and carers who have been instrumental in the focus for the group. Participation has enhanced the person's understanding of the hospice and has allowed for seamless access to Inpatient Unit/Day Therapy Unit if required, or support being put in place for home, facilitating patient choice where possible. This feedback highlights that a support group for people living with MND is valuable and beneficial to both the patient and carer, and our hope is for this service to continue at the hospice.

\section{P-192 BRINGING TOGETHER PALLIATIVE AND SPINAL INJURY CARE - A PROJECT TO SHARE AND DISSEMINATE KNOWLEDGE}

Jane Berg, Carole Cousins. Princess Alice Hospice, Esher, UK

\subsection{6/bmjspcare-2019-HUKNC.214}

Background The number of annual spinal injuries (through illness or trauma) is thought to have been underestimated. Recent data suggests that 2,500 people a year in the UK are diagnosed with a spinal injury rather than the 1,000 previously thought (Spinal Injuries Association, 2019) and the number of people living with spinal injury in the UK is 50,000 .

The type of injury is changing, the stereotypical young motorcyclist is being replaced as the most frequent patient profile by the older person who falls at home, or remains active in retirement and sustains an injury associated with an active lifestyle.

In addition, the life expectancy of people with spinal injury has improved and is now equal to that of non-injured people. Individuals are reaching old age with longstanding care needs as a result of their spinal injury.

The specialities of palliative and spinal injury care have traditionally had very different priorities but having recognised that the number of people with spinal injuries requiring palliative and end of life care is increasing, it is time to bring the two worlds together and learn from each other.

Aim The project will bring together palliative care and spinal injury care specialists to develop an education programme and resources for clinical teams caring for a person with a spinal injury who is approaching the end of life.

Method A course is being developed which will be available on-line and give easy access to information to support specialist and generalist practitioners.

A leading hospice and the Spinal Injuries Association are collaborating on the project to bring together their expertise. The presentation will outline how the project began and how the course is being developed.

Anticipated results The course will be available in Winter 2019.

This project has been funded by donation from the Tye family.

\section{P-193 KIRKLEES CARERS CONSORTIUM- PARTNERSHIP WORKING TO BENEFIT CARERS}

Catherine Thompson, Sarah Shaw, Sadaf Adnan. Kirkwood Hospice, Huddersfield, UK

\subsection{6/bmjspcare-2019-HUKNC.215}

Background The Carers Consortium is a collaboration of Kirklees carers services, which include:

- Carers Trust - a not-for-profit charity supporting carers. Offering carers breaks and homecare support;

- Carers Count - aims to promote the wellbeing of carers and support them in their caring role;

- Barnardo's- offering support for young carers aged 8-18 who support an individual affected by a physical or mental health difficulty;

- Kirklees Council;

- Kirkwood Hospice.

The individual organisations identified that there were a wide range of services in Kirklees to support carers and that there seemed to be a degree of overlap in carers' services making it confusing and complex to navigate. It was also felt that key stakeholders - including clinical staff across primary, secondary and community care - might not be aware of local services and where to signpost carers, and so a collaborative approach might support wider awareness raising and improve access to services.

As a hospice, we are extremely pleased to be recognised as a key partner in supporting carers in Kirklees. Through our involvement in the consortium, we now have the opportunity as a collective to share good practice, reduce the number of referrals that a carer would need to make and share knowledge of each others' services and areas of expertise. This integration will benefit carers in Kirklees.

A feature of our partnership working has been the development and publication of joint promotional materials, including 